\title{
High-Dose Sequential Chemotherapy and Peripheral Blood Progenitor Cell Autografting in Patients with Refractory and/or Recurrent Hodgkin Lymphoma
}

\section{A Multicenter Study of the Intergruppo Italiano Linfomi Showing Prolonged Disease Free Survival in Patients Treated at First Recurrence}

\author{
Corrado Tarella, M.D. ${ }^{1}$ \\ Alessandra Cuttica, M.D. ${ }^{1}$ \\ Umberto Vitolo, M.D. ${ }^{2}$ \\ Marina Liberati, M.D. ${ }^{3}$ \\ Massimo Di Nicola, M.D. ${ }^{4}$ \\ Sergio Cortelazzo, M.D. ${ }^{5}$ \\ Rosalba Rosato, Ph.D. ${ }^{6}$ \\ Carlo Rosanelli, M.D. ${ }^{7}$ \\ Nicola Di Renzo, M.D. ${ }^{8}$ \\ Maurizio Musso, M.D. ${ }^{9}$ \\ Enzo Pavone, M.D. ${ }^{10}$ \\ Gino Santini, M.D. ${ }^{11}$ \\ Alessandra Pescarollo, M.D. ${ }^{12}$ \\ Alberto De Crescenzo, M.D. ${ }^{13}$ \\ Massimo Federico, M.D. ${ }^{14}$ \\ Andrea Gallamini, M.D. ${ }^{15}$ \\ Patrizia Pregno, M.D. ${ }^{2}$ \\ Roberta Romano, M.D. ${ }^{3}$ \\ Paolo Coser, M.D. ${ }^{7}$ \\ Eugenio Gallo, M.D. ${ }^{2}$ \\ Mario Boccadoro, M.D. ${ }^{1}$ \\ Tiziano Barbui, M.D. ${ }^{5}$ \\ Alessandro Pileri, M.D. ${ }^{1}$ \\ Alessandro M. Gianni, M.D. ${ }^{4}$ \\ Alessandro Levis, M.D. ${ }^{16}$

\footnotetext{
${ }^{1}$ Dipartimento Medicina-Oncologia Sperimentale, Divisione Universitaria di Ematologia, Torino, Italy.

${ }^{2}$ Divisione Ospedaliera di Ematologia, Azienda 0spedaliera S. Giovanni Battista, Torino, Italy.

${ }^{3}$ Clinica Medica, Policlinico Monteluce, Perugia,
Italy.

${ }^{4}$ Divisione di Oncologia, Istituto Nazionale Tumori, Milano, Italy.
} \\ ${ }^{5}$ Divisione Ospedaliera Ematologia, Ospedaliera
Riuniti di Bergamo, Bergamo, Italy.
}

BACKGROUND. The objective of the current study was to evaluate in a multicenter setting the feasibility and efficacy of a high-dose sequential (HDS) chemotherapy regimen that combined intensive debulking and high-dose therapy (HDT) with peripheral blood progenitor cell (PBPC) autografting in patients with refractory or recurrent Hodgkin lymphoma (HL).

METHODS. Data were collected from 102 patients with HL who were treated with the HDS regimen at 14 centers associated with the Intergruppo Italiano Linfomi. Twenty-four patients had primary refractory HL, 59 patients had their first recurrence of HL (within 1 year in 32 patients and $>1$ year in 27 patients), and 19 patients had multiple disease recurrences. The HDS regimen included the sequential delivery of high-dose (hd) cyclophosphamide with PBPC harvesting, methotrexate, etoposide, then HDT (usually hd mitoxantrone plus L-phenylalanine mustard) with PBPC autografting. In addition, radiotherapy was delivered to 36 patients at sites of bulky or persistent disease.

RESULTS. Ninety-two patients $(90 \%)$ completed the HDS program. There were five toxic deaths (treatment-related mortality rate, $4.9 \%$ ) and six secondary malignan-

\footnotetext{
${ }^{6}$ Servizio di Epidemiologia dei Tumori, Università di Torino, Torino, Italy.

${ }^{7}$ Divisione di Ematologia, Azienda Ospedaliera S. Maurizio, Bolzano, Italy.

${ }^{8}$ Divisione di Ematologia, Azienda Ospedaliera Casa Sollievo della Sofferenza, S. Giovanni Rotondo, Italy.

${ }^{9}$ Divisione di Ematologia, Universitaria di Palermo, Palmero, Italy.

${ }^{10}$ Divisione di Ematologia, Universitaria di Bari, Bari, Italy.

${ }^{11}$ Dipartimento di Ematologia, Azienda Osped-
aliera S. Martino, Genova, Italy.

${ }^{12}$ Bone Marrow Transplantation Unit, Istituto Scientifico H. S. Raffaele, Milano, Italy.

${ }^{13}$ Divisione di Medicina Generale, Ospedale S. Giovanni Antica Sede, Torino, Italy.
${ }^{14}$ Divisione di Oncologia, Universitaria di Modena, Modena, Italy.

\footnotetext{
${ }^{15}$ Divisione di Ematologia, Azienda Ospedaliera S. Croce, Cuneo, Italy.

${ }^{16}$ Divisione di Ematologia, Azienda Ospedaliera SS. Antonio e Biagio, Alessandria, Italy.
}

Supported in part by Associazione Italiana Ricerca sul Cancro, Milan, Italy and by Compagnia di San Paolo, Torino, Italy.

The authors thank the President, Prof. L. Resegotti; the National Coordinator, Dr. Maura Brugiatelli; and all Centers of the Intergruppo Italiano Linfomi for their invaluable help and collaboration in designing the study and collecting the data.

Address for reprints: Corrado Tarella, Divisione Universitaria di Ematologia, Azienda Ospedaliera S. Giovanni Battista, Via Genova 3, Torino 10126, Italy; Fax: (011) 39 0116963737; E-mail: corrado.tarella@unito.it

Received November 4, 2002; revision received February 3, 2003; accepted February 4, 2003. 
cies (five patients developed myelodysplastic syndrome/acute myelogenous leukemia, and one patient developed colorectal carcinoma). At a median follow-up of 5 years, the 5-year overall survival (OS) and event-free survival (EFS) projections were $64 \%$ (95\% confidence interval [95\% CI], 54-74\%) and 53\% (95\% CI, 43-63\%), respectively. Patients with their first recurrence had the most favorable outcome, with 5-year OS and EFS projections of $77 \%$ (95\% CI, 66-88\%) and $63 \%$ (95\% CI, $50-76 \%)$, respectively. There were no significant differences between patients with early first recurrence and late first recurrence. The poorest outcome was observed in patients with refractory HL, with 5-year OS and EFS projections of 36\% (95\% CI, $16-55 \%)$ and $33 \%$ (95\% CI, 14-52\%), respectively. Patients who received HDS chemotherapy after multiple recurrences had an intermediate outcome. Multivariate analysis showed that refractory disease and systemic symptoms at the time of initial presentation were associated significantly associated with poor OS and EFS. CONCLUSIONS. The use of HDS chemotherapy for patients with refractory and/or recurrent $\mathrm{HL}$ is feasible at the multicenter level. The combination of intensive debulking and HDT with PBPC autografting offers a good chance of prolonged disease free survival for patients with their first recurrence of HL. Cancer 2003;97: 2748-59. (C) 2003 American Cancer Society.

DOI 10.1002/cncr.11414

KEYWORDS: Hodgkin Iymphoma, high-dose chemotherapy, peripheral blood progenitor cell autograft, clinical outcome, multicenter trial.

H igh-dose therapy (HDT) plus autotransplantation is now a widely employed procedure for the treatment of patients with refractory or recurrent Hodgkin lymphoma (HL) after they receive radiotherapy or conventional-dose chemotherapy. ${ }^{1-3}$ One randomized study and several nonrandomized, single-institution studies showed that these patients had a better outcome after HDT compared with patients who received conventional-dose treatments. ${ }^{4-10}$ Those findings are supported further by registry-based, retrospective studies. ${ }^{11-14}$

The applicability and efficacy of HDT have been improved by autografting mobilized peripheral blood progenitor cells (PBPCs), ${ }^{15,16}$ and preliminary results indicate that this also is beneficial for patients with HL. ${ }^{17}$ In addition, intensive, prior chemotherapy debulking seems to increase the probability of long-term survival. A recent two-step, comprehensive program that includes intensive debulking, PBPC collection, and final HDT with autotransplantation has been employed successfully in patients with recurrent and/or refractory HL. ${ }^{18}$ Patients entering that program had a long-term survival projection of $73 \%$, which compares favorably with the results reported to date with HDT. A similar approach with debulking chemotherapy followed by autotransplantation was employed by the French Lymphoma Study Group and yielded satisfactory results in patients who completed the program. ${ }^{19}$

The high-dose sequential (HDS) chemotherapy regimen is a comprehensive approach that includes an intensified debulking phase through the sequential administration of high-dose (hd) cyclophosphamide, methotrexate, and etoposide followed by autograft. ${ }^{15,20}$ The scheme takes advantage of the usually large amounts of PBPCs that can be collected after hd cyclophosphamide. ${ }^{15,21}$ HDS chemotherapy was developed several years ago by the Milan group, who conducted a single-center study in patients with HL who failed to respond to conventional therapy. ${ }^{22}$ HDS chemotherapy was effective, particularly in patients with their first episode of recurrent disease..$^{22,23}$

The original HDS regimen and its modified versions have been employed widely in the last decade in Italy for the management of both patients with HL and patients with non-Hodgkin lymphoma. ${ }^{24-28}$ This prompted us to undertake a retrospective assessment of the use of HDS chemotherapy as salvage therapy for patients with $\mathrm{HL}$ at centers that belong to the Intergruppo Italiano Linfomi (IIL) organization. The objectives of this study were to evaluate 1) the applicability of HDS chemotherapy in a multicenter setting, 2) its tolerability and efficacy in patients with HL who fail to respond to conventional therapy, and 3) differences in outcome according to disease status at the time patients received salvage treatment.

\section{MATERIALS AND METHODS Patients}

The IIL is an Italian organization that was set up to coordinate and collect data from multicenter study groups working in the field of lymphoma. It has recently collected retrospective data from 14 Italian cen- 
ters on 102 patients who received the original HDS regimen as salvage treatment for refractory or recurrent HL between January 1993 and September 2000. Six centers contributed to the study by reporting the majority of patients (83 of 102 patients; $81 \%$ ), and 8 smaller centers reported the remaining 19 patients. The common eligibility criteria were age younger than 60 years; no major organ dysfunction for causes unrelated to HL, including 1) normal cardiac function (systolic ejection fraction $>50 \%$ ), 2) no chronic respiratory disease, 3) no more than 2.5 times the upper limit of normal for liver and renal function tests, 4) no psychiatric disease, and 5) a negative human immunodeficiency virus test; and the ability to provide written, informed consent, which was provided by all patients before they started the program. A poor performance status or failure to respond to conventional chemotherapy were not exclusion criteria. In 11 centers that reported 98 of 102 patients, HDS chemotherapy was the standard treatment for all patients with HL who were younger than 60 years and presented with refractory or recurrent disease.

Disease persistence or recurrence was proven by biopsy or fine-needle aspiration biopsy (57 patients) or was demonstrated unequivocally on radiographic studies. Refractory disease was defined as stable or progressive disease or a tumor reduction $<50 \%$, and partial remission (PR) was defined as a reduction $>50 \%$ in the greatest tumor dimension for all known sites with no new disease sites after completion of the induction treatment. Recurrence was defined as recurrent HL after a documented complete remission (CR) that lasted at least 1 month after induction.

Overall, 24 patients had refractory disease after first-line therapy; 59 patients had their first recurrence (32 patients within the first 12 months after first-line therapy and 27 patients $>12$ months after first-line therapy: median, 24 months; range, 13-156 months) after achieving CR. The other 19 patients received HDS chemotherapy after multiple recurrences. According to the Revised European-American classification of lymphoid neoplasms, the histologic subtypes included nodular sclerosis (68.5\%), mixed cellularity (19\%), lymphocyte depletion (7.5\%), and lymphocyte predominance $(5 \%){ }^{29}$

All patients had their disease staged according to the Cotswold modification of the Ann Arbor system. ${ }^{30}$ Their main characteristics at the time they started the HDS regimen, along with their previous treatments, are summarized in Table 1. Most patients had received either doxorubicin, bleomycin, vinblastine, and dacarbazine (ABVD) or multiple-drug regimens, such as nitrogen mustard, vincristine, procarbazine, and prednisone (MOPP) plus ABVD or MOPP plus epirubicin, bleomycin, and vincristine plus cyclophosphamide, doxorubicin, and dacarbazine (EBV/CAD) ${ }^{31-34}$

\section{Treatment Plan}

The HDS regimen is a comprehensive program that includes a high-dose (hd) chemotherapy phase with peripheral blood progenitor cell (PBPC) harvesting and a final myeloablative treatment with PBPC autografting. ${ }^{15,20}$ The hd phase includes the sequential administration at 10-day to 15-day intervals of hd cyclophosphamide (CY; $7 \mathrm{~g} / \mathrm{m}^{2}$ ), hd methotrexate (8 $\mathrm{g} / \mathrm{m}^{2}$ ), and hd etoposide (VP16; $2 \mathrm{~g} / \mathrm{m}^{2}$ ); granulocytecolony stimulating factor is given daily at a dose of 5 $\mathrm{mcg} / \mathrm{kg}$ after both CY and VP16 to reduce hematologic toxicity and to expand the circulating progenitor cell pool. PBPC harvesting is scheduled after CY, and a second harvest is performed after VP16 in patients who have inadequate PBPC collection after they receive CY. Patients with bone marrow (BM) involvement receive a modified schedule with inversion of the CY/VP16 sequence and PBPC collection at the end of the hd phase; this modification was designed to exploit a kind of in vivo purging effect from chemotherapy prior to harvests.

Ninety-two of 102 patients $(90 \%)$ completed the program with the final myeloablative treatment and the autograft. The median time between the beginning of sequential chemotherapy and the autograft was 3 months (range, 2-8 months). Seventy-nine patients (86\%) were conditioned with the mitoxantrone/L-phenylalanine mustard (L-PAM) combination (i.e., mitoxantrone $60 \mathrm{mg} / \mathrm{m}^{2}$ on Day 5, L-PAM $180 \mathrm{mg} / \mathrm{m}^{2}$ on Day 2, and autograft on Day 0). ${ }^{35}$ Thirteen patients (14\%) received other regimens: carmustine, etoposide, cytarabine, and melphalan (BEAM; $n=10$ patients); L-PAM $180 \mathrm{mg} / \mathrm{m}$ only ( $n=2$ patients); or thiotepa/ L-PAM ( $n=1$ patient). ${ }^{5}$ The decision to use conditioning regimens other than the mitoxantrone/L-PAM combination was made at the discretion of treating physicians in the different centers based on the expertise of each center on a given scheme and in the absence of any documented superiority of one regimen over the others.

All patients had an indwelling central venous catheter. The entire pretransplantation program was carried out in ordinary, unprotected rooms. Autografting was conducted in a dedicated inpatient BM transplantation unit. During pancytopenia and after autografting, patients were managed with a common protocol for prophylaxis comprised of oral antibiotics (usually ciprofloxacin $500 \mathrm{mg}$ three times daily, fluconazole $150 \mathrm{mg}$ intravenously, and acyclovir $250 \mathrm{mg}$. three times daily). In the event patients developed fever $>38{ }^{\circ} \mathrm{C}$, blood cultures and chest X-rays were 
TABLE 1

Main Characteristics of Patients who Received High-Dose Sequential Chemotherapy According to Disease Status Subgroups

\begin{tabular}{|c|c|c|c|c|c|}
\hline \multirow[b]{3}{*}{ Parameter } & \multicolumn{4}{|c|}{ Patient subgroup } & \multirow[b]{3}{*}{ Total (\%) } \\
\hline & \multirow[b]{2}{*}{ Refractory } & \multicolumn{2}{|c|}{ First recurrence } & \multirow[b]{2}{*}{$\begin{array}{l}\text { Later than } \\
\text { first recurrence }\end{array}$} & \\
\hline & & $\begin{array}{l}\mathrm{CR} \leq 12 \\
\text { months }\end{array}$ & $\begin{array}{l}\text { CR }>12 \\
\text { months }\end{array}$ & & \\
\hline No. of patients & 24 & 32 & 27 & 19 & 102 \\
\hline \multicolumn{6}{|l|}{ Gender } \\
\hline Male & 8 & 15 & 20 & 12 & $55(54)$ \\
\hline Female & 16 & 17 & 7 & 7 & $47(46)$ \\
\hline \multicolumn{6}{|l|}{ Age (yrs) } \\
\hline Median (range) & 27 & 27 & 36 & 37 & 32 \\
\hline Range & $13-60$ & $16-59$ & $18-60$ & $17-58$ & $13-60$ \\
\hline \multicolumn{6}{|l|}{ Ann Arbor stage } \\
\hline I-II & 13 & 13 & 12 & 4 & $42(41)$ \\
\hline III-IV & 11 & 19 & 15 & 15 & $60(59)$ \\
\hline B symptoms & 15 & 8 & 8 & 12 & $43(42)$ \\
\hline Elevated LDH level & 7 & 6 & 2 & 4 & $19(19)$ \\
\hline BM involvement ${ }^{\mathrm{a}}$ & 0 & 3 & 5 & 3 & $11(11)$ \\
\hline Bulky disease $^{\mathrm{b}}$ & 12 & 8 & 4 & 6 & $30(29)$ \\
\hline Performance status $\geq 2^{\mathrm{c}}$ & 6 & 4 & 1 & 3 & $14(14)$ \\
\hline \multicolumn{6}{|l|}{ No. of extralymph node sites } \\
\hline None & 16 & 22 & 14 & 10 & $62(61)$ \\
\hline 1 & 7 & 7 & 8 & 8 & $30(29)$ \\
\hline$\geq 2$ & 1 & 3 & 5 & 1 & $10(10)$ \\
\hline Previous RT & 4 & 18 & 16 & 11 & $49(48)$ \\
\hline \multicolumn{6}{|l|}{ First-line therapy } \\
\hline ABVD & 12 & 8 & 6 & 2 & 28 (27) \\
\hline MOPP & 0 & 0 & 0 & 1 & $1(1)$ \\
\hline Seven or eight-drug regimen & 12 & 22 & 14 & 10 & $58(57)$ \\
\hline Other protocols & 0 & 2 & 4 & 5 & $11(11)$ \\
\hline RT only & 0 & 0 & 3 & 1 & $4(4)$ \\
\hline
\end{tabular}

taken, and patients were treated empirically with intravenous, broad-spectrum antibiotics. Vancomicin was added only if a surveillance culture documented the presence of methicillin-resistant, gram positive cocci; if the fever was of undetermined origin and persisted after 36-48 hours, then intravenous imipenem was introduced, and intravenous amphotericin was added if the fever persisted for another $36-48$ hours. Antibiotics were continued until the temperature reverted to normal for at least 48 hours and the absolute neutrophil count to reverted $>500 / \mu \mathrm{L}$. Irradiated, leukocyte-filtered, single-donor platelet concentrates or, less frequently, multiple-donor, irradiated platelet concentrates were given to patients with platelet counts $<20,000 / \mu \mathrm{L}$; and irradiated, leukocyte-filtered, packed red blood cells were given to patients with hemoglobin levels $<8 \mathrm{~g} / \mathrm{dL}$. Prophylaxis with cotrimoxazole twice per week plus acyclovir was maintained for 6 months after the autograft.

Radiotherapy was scheduled for patients with sites of bulky or persistent disease approximately 2 months after autograft. Overall, 36 patients received radiotherapy, including 35 patients who received radiotherapy as postautograft consolidation and 1 patient who received radiotherapy for debulking prior to autograft.

\section{Response and Long-Term Outcome Assessment}

Patients had their disease restaged once before the autograft and again within 2 months after HDS chemotherapy using common laboratory tests, radiologic examinations, and BM biopsy. A restaging procedure was scheduled every 6 months for the first 3 years and annually thereafter. Computed tomography (CT) 
TABLE 2

Response to High-Dose Sequential Chemotherapy Program According to Disease Status Subgroups

\begin{tabular}{|c|c|c|c|c|c|}
\hline \multirow[b]{3}{*}{ Parameter $^{\mathrm{a}}$} & \multicolumn{4}{|c|}{ Patient subgroup: No. of patients (\%) } & \multirow[b]{3}{*}{ Total (\%) } \\
\hline & \multirow[b]{2}{*}{ Refractory } & \multicolumn{2}{|c|}{ First recurrence } & \multirow[b]{2}{*}{$\begin{array}{l}\text { Later than } \\
\text { first recurrence }\end{array}$} & \\
\hline & & $\begin{array}{l}\mathrm{CR} \leq 12 \\
\text { months }\end{array}$ & $\begin{array}{l}\text { CR }>12 \\
\text { months }\end{array}$ & & \\
\hline No. of patients & 24 & 32 & 27 & 19 & 102 \\
\hline HDS completion & $21(87)$ & $30(94)$ & $24(89)$ & $17(89)$ & $92(90)$ \\
\hline $\mathrm{CR}$ & $10(42)$ & $25(78)$ & $24(89)$ & $14(74)$ & $73(71.5)^{\mathrm{b}}$ \\
\hline $\mathrm{PR}$ & $5(21)$ & $2(6)$ & $2(7)$ & $2(11)$ & $11(10.8)$ \\
\hline NR or progression & $7(29)$ & $4(13)$ & $0(0)$ & $2(11)$ & $13(12.7)$ \\
\hline Toxic death ${ }^{\mathrm{c}}$ & $2(8)$ & $1(3)$ & $1(4)$ & $1(5)$ & $5(4.9)$ \\
\hline
\end{tabular}

scans of the chest and abdomen were performed routinely, and a second radiologic assessment (e.g., Gallium scan or nuclear magnetic resonance imaging) was done only in patients who had a residual, suspicious mass on CT scan. BM biopsy was repeated only for patients with previously detected BM disease involvement. The response criteria are described above. The two patients who were diagnosed with an undefined CR at the end of HDS chemotherapy were included either among the CR group or the PR group, depending on their status at the first subsequent restaging.

\section{Statistical Analysis}

All patients were evaluated. The proportions of patients with given characteristics were compared among subgroups, with statistically significance differences tested using the Fisher exact test. In addition, the influence of disease status on treatment feasibility and on the achievement of CR was evaluated with the Fisher exact test.

Follow-up was updated in December 2002, and all living patients had been observed at least once in the previous 6 months. Overall survival (OS) was calculated from the start of HDS chemotherapy to the date of death or last follow-up. Event-free survival (EFS) was measured from the start of HDS chemotherapy until the first event (death from any cause, recurrence, or occurrence of a second neoplasm) or last follow-up. The actuarial durations of OS and EFS were plotted as curves according to the Kaplan-Meier product-limit method. ${ }^{36}$ Differences in OS and EFS according to disease status were evaluated, including refractory disease versus recurrent disease (refractory disease vs. first recurrence vs. later than first recurrence) and the duration of first CR (12 months vs. $>12$ months). Differences in OS also were evaluated according to whether patients achieved CR after the hd phase, prior to autograft. Differences in survival between groups were identified using a generalized log-rank analysis. ${ }^{37}$ All $P$ values were two-sided, and the cut-off value for significance was $P<0.05$. Several clinical variables at the time patients received HDS chemotherapy were evaluated in a univariate analysis for possible prognostic significance, including age (younger than 40 years or older than 40 years), gender, disease stage, B symptoms, BM involvement, bulky disease, extralymph node sites, prior radiotherapy, and disease status. Patients also were evaluated in a multivariate analysis using a stepwise Cox regression model. ${ }^{38}$

\section{RESULTS}

\section{Feasibility and Response}

The HDS schedule was feasible at the multicenter level. Table 2 shows that 92 of 102 patients $(90 \%)$ completed the program. Interruptions were due to 1) no response or disease progression in six patients, 2) insufficient PBPC or BM harvests in two patients, 3) pulmonary mycotic infection one patient, and 4) patient refusal in one patient. There were no significant differences in feasibility among the disease status subgroups, as shown in Table 2.

PBPC mobilization was evaluable in 101 patients: It was satisfactory $\left(>2 \times 10^{6}\right.$ harvested CD34 positive cells per $\mathrm{kg}$ ) in 89 patients (88\%), low in 8 patients, and absent in 4 patients. Overall, the median value of harvested CD34 positive cells per $\mathrm{kg}$ was $6.9 \times 10^{6}$ cells $/ \mathrm{kg}$ (range, 1-69 cells $/ \mathrm{kg}$ ). Autograft was per- 
formed with PBPCs in 82 patients (80.4\%), with PBPCs and BM cells in 8 patients (7.8\%), and with BM cells in 2 patients $(1.9 \%)$.

All patients were evaluated for response and longterm outcome in an intent-to-treat analysis. Details of the response to HDS chemotherapy are reported in Table 2. Overall, 73 patients (72\%) achieved CR, and 24 patients (23.6\%) had a poor response, including PR, no response, and disease progression. Patients with refractory disease had a significantly lower CR rate $(42 \%)$ compared with patients in the other subgroups (7489\%; $P=0.0022$ ).

Overall, 69 patients (68\%) already had achieved CR by the end of chemotherapy, and 4 more patients reached CR after consolidation radiotherapy. Only two patients were categorized with an undefined CR at the end of the program: One patient had a stable CR and was included in the CR group, and the second patient had early disease progression at 5 months after receiving HDS chemotherapy and was included in the $\mathrm{PR} /$ no response group.

\section{Toxicity}

There were five toxic deaths (Table 2), which were due to heart failure in one patient 8 months after autograft and to pneumonia in four patients that was caused by aspergillosis in two patients, cytomegalovirus in one patient, and an unidentified agent in the fourth patient. These infections manifested during the hd phase in two patients and occurred 1 month and 5 months after autograft in the other two patients.

Hematopoietic recovery and transfusion requirements after hd CY, hd VP16, and autograft are summarized in Table 3 . There were some episodes of Grade 3-4 extrahematologic, early, nonfatal toxicity (other than oral and gastrointestinal mucositis during the myeloablative phase), including 10 episodes of pneumonia; 5 episodes of liver toxicity, 14 septic episodes, 2 episodes of hemorrhagic cystitis, 1 episode of supraventricular tachyarrhythmia, 1 episode of atrial fibrillation, and 1 episode of neurologic toxicity. Overall, $55 \%$ of these episodes were recorded during the debulking hd phase, and $45 \%$ of episodes were recorded during the final myeloablative phase. No differences in toxicity were observed between the subgroups.

Finally, 6 patients developed a secondary malignancy: Four of them developed refractory anemia with blast excess at 13 months, 20 months, 24 months, and 26 months after the completion of HDS chemotherapy; and 1 patient developed acute myeloblastic leukemia (AML) 6 months after autograft. Four of these patients received HDS chemotherapy for either primary refractory disease (one patient) or first recur-
TABLE 3

Hematologic Toxicity during Delivery of High-Dose Sequential Chemotherapy

\begin{tabular}{|c|c|c|c|}
\hline \multirow[b]{2}{*}{ Parameter } & \multicolumn{3}{|c|}{ Median (range) } \\
\hline & $\begin{array}{l}\text { After } \\
\mathrm{CY} 7 \mathrm{~g} / \mathrm{m}^{2}\end{array}$ & $\begin{array}{l}\text { After } \\
\text { VP16 } 2 \mathrm{~g} / \mathrm{m}^{2}\end{array}$ & Autograft \\
\hline No. of patients & 80 & 80 & 92 \\
\hline $\begin{array}{l}\text { Days with ANC }<500 \mathrm{~mL} \\
\text { RBC Tx }\end{array}$ & $5(0-11)$ & $5(0-10)$ & $8(5-25)$ \\
\hline Patients receiving Tx (\%) & 42 & 37 & 93 \\
\hline No. of units received & $2(1-6)$ & $2(1-6)$ & $3(1-10)$ \\
\hline Plts Tx & & & \\
\hline Patients receiving Tx (\%) & 36 & 16 & 98 \\
\hline No. of units received & $2(1-11)$ & $2(1-38)$ & $2(1-24)$ \\
\hline Patients with fever (\%) & 39 & 47 & 83 \\
\hline Patients with severe & & & \\
\hline infections $(\%)^{\mathrm{a}}$ & 8 & 6 & 14 \\
\hline $\begin{array}{l}\text { Patients with Grade 3-4 } \\
\text { mucositis (\%) }\end{array}$ & 3 & 2 & 32 \\
\hline
\end{tabular}

CY: cyclophosphamide; VP16: etoposide: ANC: absolute neutrophil count; RBC TX: red blood cell transfusion; Plts Tx: platelet transfusion.

${ }^{a}$ Severe infections included 10 patients with of pneumonia, 16 septic episodes, and 2 patients with hemorrhagic cystitis.

rence (three patients); the fifth patient received HDS chemotherapy after a third recurrence and multiple chemoradiotherapy treatments. One other patient, who received HDS chemotherapy after his second recurrence, developed colorectal carcinoma 6 years after HDS chemotherapy. All patients who developed secondary malignancies had been exposed to radiotherapy either as part of their first-line treatment or at the time they were diagnosed with recurrent disease. In addition, four patients received further treatment after HDS chemotherapy due to disease recurrence. Four of these six patients died of complications related to their secondary malignancy.

\section{Long-Term Outcome}

At the closing date of this analysis, 63 patients were alive (54 patients without signs of disease or severe treatment-related complications), 36 patients had died, and 3 patients had been lost to follow-up. Other than the 5 treatment-related fatalities, 26 deaths were related to disease progression, 4 deaths were related to secondary malignancies, and there was 1 more late death after 3 years due to unknown causes. At a median follow-up of 5 years, the actuarial OS and EFS curves for the entire group had 5-year projections of $64 \%$ (95\% confidence interval [95\% CI], 54-74\%) and $53 \%$ (95\% CI, 43-63\%), respectively.

Long-term outcome was evaluated according to disease status at the start of HDS chemotherapy. The 


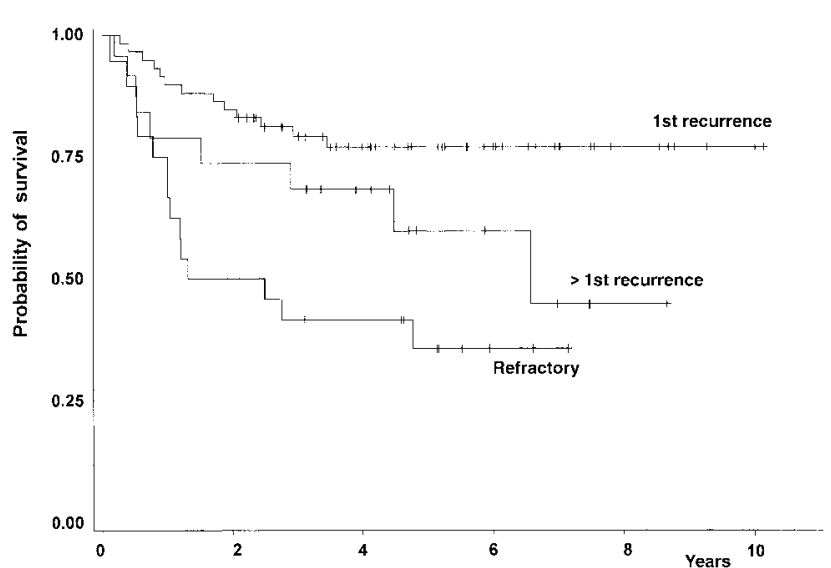

FIGURE 1. Estimated overall survival according to disease status at the time patients receive high-dose sequential chemotherapy. Patients with refractory disease had a significantly poorer outcome compared with patients in the two other main subgroups (patients with their first episode of recurrent disease and patients with two or more episodes of recurrent disease; $P=0.003$ )

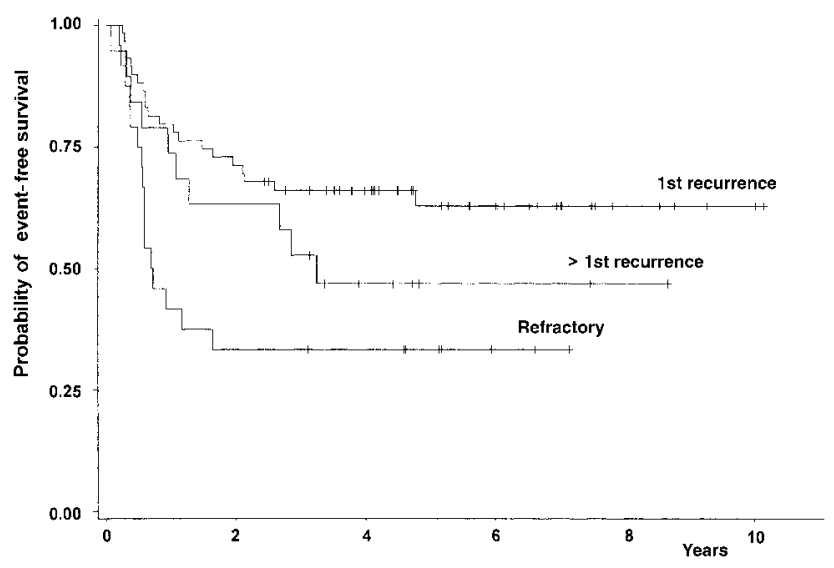

FIGURE 2. Estimated event-free survival according to disease status at the time patients received high-dose sequential chemotherapy. Patients with refractory disease had a significantly poorer outcome compared with patients in the two other main subgroups (patients with their first episode of recurrent disease and patients with two or more episodes of recurrent disease; $P$ $=0.009$ )

median follow-up for all subgroups varied between 4.7 years and 5.3 years. The actuarial OS and EFS curves for the three main subgroups (patients with refractory disease, patients with their first episode of recurrent disease, and patients with two or more episodes of recurrent disease) are shown in Figures 1 and 2. The 5 -year OS projections were $36 \%$ (95\% CI, $16-55 \%$ ) for patients with refractory disease, $77 \%$ (95\% CI, 66-88) for patients with their first episode of recurrent disease, and 59\% (95\% CI, 36-84\%) for patients with $\geq 2$ episodes of recurrent disease. The 5-year EFS projections were $33 \%(95 \% \mathrm{CI}, 14-52 \%)$ for patients with

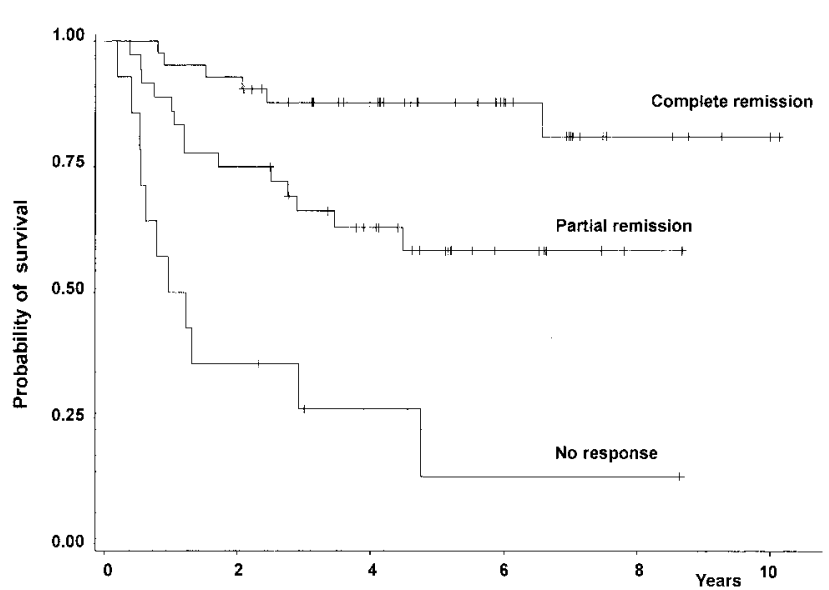

FIGURE 3. Estimated overall survival according to disease status after patients received high-dose chemotherapy prior to autograft comparing patients who achieved complete remission ( $n=42$ patients) with patients who achieved partial remission ( $n=36$ patients) and patients who had no response ( $n=14$ patients). Patients who achieved complete remission had a significantly better outcome compared with patients who achieved partial remission $(P=0.01)$ and patients who had no response $(P=0.0002)$.

refractory disease, $63 \%$ (95\% CI, 50-76\%) for patients with their first episode of recurrent disease, and $47 \%$ (95\% CI, 24-69\%) for patients with $\geq 2$ episodes of recurrent disease. Patients with refractory disease had a significantly poorer outcome in terms of both OS ( $P$ $=0.003)$ and EFS $(P=0.009)$ compared with the other two main subgroups.

There were no significant differences between patients who were treated for early (within 1 year) or late ( $>1$ year) first recurrence. The respective actuarial 5 -year OS projections were $77 \%$ (95\% CI, 62-92\%) and $77 \%$ (95\% CI, 60-93\%), respectively; and the actuarial 5 -year EFS projections were $69 \%$ (95\% CI, 52-85\%) and $56 \%$ (95\% CI, 35-77\%).

Finally, long-term outcome was evaluated according to disease status after hd chemotherapy prior to autograft, i.e., patients who achieved CR $(n=42$ patients) and patients who did not achieve CR, including 36 patients who had a PR and 14 patients who had no response. Figure 3 shows that significant differences were observed in the OS curves, with actuarial 5-year projections of $88 \%$ (95\% CI, $65-97 \%$ ) for patients who achieved CR compared with 58\% (95\% CI, 41-76\%) for patients who had a PR $(P=0.01)$ and $13 \%(95 \% \mathrm{CI}$, $0-36 \%)$ for patients who had no response $(P=0.0002)$. Analogous differences were observed in the EFS, with 5 -year projections of $78 \%$ (95\% CI, 65-91\%) for patients who achieved CR, $41 \%$ (95\% CI, 23-59\%) for patients who had a PR $(P=0.0007)$, and $7 \%(95 \% \mathrm{CI}$, $0-20 \%)$ for patients who had no response $(P<0.0001)$, as shown in Figure 4. No significant differences were 


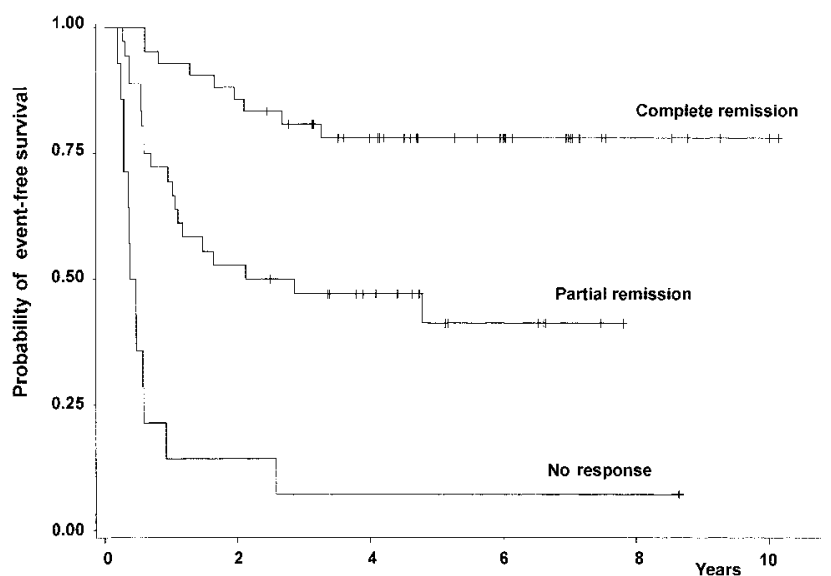

FIGURE 4. Estimated event-free survival according to disease status after patients received high-dose chemotherapy prior to autograft comparing patients who achieved complete remission ( $n=42$ patients) with patients who achieved partial remission ( $n=36$ patients) or had no response $(n=14$ patients). Patients who achieved complete remission had a significantly better outcome compared with patients who achieved partial remission $(P=0.0007)$ and patients who had no response $(P<0.0001)$.

observed between patients who received autografts after mitoxantrone/L-PAM and patients who received other conditioning regimens.

\section{Prognostic Parameters}

Several clinical characteristics at the time patients received HDS chemotherapy were evaluated for their prognostic value on OS and EFS in a univariate analysis. Table 4 shows that two variables-primary refractory disease and B symptoms - at the time patients started HDS chemotherapy were associated with poor OS and EFS. These two variables had independent, adverse prognostic value in a multivariate analysis (Table 5).

\section{DISCUSSION}

HDT with autograft is considered the treatment of choice for patients younger than 60 years with HL who experience primary failure after conventional induction therapy. A debulking treatment usually is delivered prior to autograft. Indeed, a status in remission before autografting is regarded as a very favorable prognostic factor. However, it remains uncertain whether short or prolonged debulking therapy and conventional or intensive debulking therapy should be included in the salvage program. To address this issue, the current retrospective study was conducted based on data provided by 14 Italian centers using the HDS regimen as rescue therapy for patients with HL. This well-established regimen is comprised of intensive de-
TABLE 4

Univariate Analysis

\begin{tabular}{|c|c|c|c|c|c|}
\hline Parameter ${ }^{\mathrm{a}}$ & $\begin{array}{l}\text { No. of } \\
\text { patients }\end{array}$ & $\begin{array}{l}\text { Three-yr } \\
\text { OS } \\
\text { projection } \\
(\%)\end{array}$ & $P$ value & $\begin{array}{l}\text { Three-yr } \\
\text { EFS } \\
\text { projection } \\
(\%)\end{array}$ & $P$ value \\
\hline \multicolumn{6}{|l|}{ Age } \\
\hline$<40$ yrs & 72 & 67 & - & 58 & - \\
\hline$>40$ yrs & 29 & 68 & 0.43 & 48 & 0.58 \\
\hline \multicolumn{6}{|l|}{ Gender } \\
\hline Female & 46 & 65 & - & 54 & - \\
\hline Male & 56 & 70 & 0.62 & 56 & 0.61 \\
\hline \multicolumn{6}{|l|}{ Stage } \\
\hline I-II & 41 & 59 & - & 53 & - \\
\hline III-IV & 60 & 73 & 0.55 & 56 & 0.89 \\
\hline \multicolumn{6}{|l|}{ BM involvement } \\
\hline No & 90 & 68 & - & 56 & - \\
\hline Yes & 11 & 63 & 0.12 & 45 & 0.41 \\
\hline \multicolumn{6}{|l|}{ Bulky disease } \\
\hline No & 71 & 71 & - & 58 & - \\
\hline Yes & 30 & 58 & 0.30 & 51 & 0.63 \\
\hline \multicolumn{6}{|c|}{ Extralymph node involvement } \\
\hline No & 60 & 69 & - & 61 & - \\
\hline Yes & 41 & 65 & 0.13 & 46 & 0.16 \\
\hline \multicolumn{6}{|l|}{ Previous radiotherapy } \\
\hline No & 53 & 65 & - & 54 & - \\
\hline Yes & 49 & 70 & 0.60 & 56 & 0.86 \\
\hline \multicolumn{6}{|l|}{ B symptoms } \\
\hline No & 58 & 82 & - & 70 & - \\
\hline Yes & 43 & 48 & 0.0001 & 34 & 0.0001 \\
\hline \multicolumn{6}{|l|}{ Disease status } \\
\hline First recurrence & 58 & 79 & - & 65 & - \\
\hline$>$ First recurrence & 19 & 68 & - & 52 & - \\
\hline Refractory & 24 & 41 & 0.003 & 33 & $0.01^{\mathrm{b}}$ \\
\hline
\end{tabular}

OS: overall survival; EFS: event free survival; BM: bone marrow.

${ }^{a}$ Parameters include clinical variables at the time patients received high-dose, sequential chemotherapy.

${ }^{\mathrm{b}}$ Patients with refractory disease versus patients in first recurrence and patients later than first recurrence.

bulking with sequential hd drug delivery followed by HDT with PBPC autografting. The results of the current study demonstrate 1) the feasibility of using HDS chemotherapy in a multicenter setting; 2) a high CR rate and prolonged OS; and 3) a definitely satisfactory outcome for patients with their first episode of recurrent disease, without significant differences between patients with early or late first recurrence.

The current analysis was performed on 102 patients who were treated with HDS chemotherapy from 1993 to 2000. Although HDS chemotherapy was the standard treatment for the rescue of patients with refractory/recurrent HL in most participating centers, a selection bias in the patient population cannot be ruled out. However, the most substantial risk factors, such as advanced disease stage, systemic symptoms, and extralymph node disease at salvage therapy, oc- 
TABLE 5

Relative Risk in Multivariate Analysis

\begin{tabular}{|c|c|c|c|c|c|c|}
\hline \multirow[b]{2}{*}{ Characteristic } & \multicolumn{2}{|c|}{ OS as endpoint } & \multirow[b]{2}{*}{$P$ value } & \multicolumn{2}{|c|}{$\begin{array}{c}\text { EFS as } \\
\text { endpoint }\end{array}$} & \multirow[b]{2}{*}{$P$ value } \\
\hline & RR & $95 \%$ CI & & RR & $95 \% \mathrm{CI}$ & \\
\hline \multicolumn{7}{|l|}{ Status at HDS } \\
\hline Refractory & 3.80 & $1.4-10.0$ & 0.008 & 2.47 & $1.1-5.5$ & 0.03 \\
\hline B symptoms & 3.89 & $1.7-8.9$ & 0.001 & 2.76 & $1.4-5.4$ & 0.003 \\
\hline
\end{tabular}

OS: overall survival; EFS: event free survival: RR: risk ratio; 95\% CI: 95\% confidence interval; HDS: high-dose sequential chemotherapy.

curred with the same frequency reported in other studies on HDT. In addition, most patients had received highly effective chemotherapy regimens prior to salvage HDS chemotherapy, including ABVD or other multiple-drug combination schemes. ${ }^{31-34}$ Thus, our patients had the clinical presentation observed commonly in patients with refractory or recurrent HL. ${ }^{39}$

The feasibility of HDS chemotherapy was among the primary endpoints of the study. Indeed, the regimen proved to be feasible, with $90 \%$ of patients completing the whole program. In addition, most patients were able to mobilize and collect PBPC amounts adequate for autografting, and only 4 of 101 evaluable patients showed no sign of mobilization. It is now a common policy to use blood-derived hemopoietic progenitors for autotransplantation in the treatment of patients with HL. However, few single-center studies have detailed the extent of PBPC mobilization in patients with HL who were not pretreated heavily. ${ }^{17,18}$ Our results are in line with those reports and confirm that PBPC mobilization and collection as part of the treatment of patients with refractory or recurrent HL is an easily reproducible approach.

HDS chemotherapy also had good tolerability. There were five treatment-related deaths, four deaths from complications related to secondary malignancies, and one death due to unspecified causes 2-6 years after HDS chemotherapy. Even when those five events were included among late toxic events, the overall treatment-related mortality (TRM) rate was on the order of $9.8 \%$. This is in line with the 9-15\% TRM rates reported by the Autologous Blood and Marrow Transplant Registry and in other retrospective, multicenter studies. ${ }^{13,14,19}$ The incidence of secondary malignancies was almost within the commonly expected range, with five patients developing myelodysplastic syndrome/AML and one patient developing colorectal carcinoma. In fact, an overall incidence rate for secondary tumors ranging between $4 \%$ and $8 \%$ has been reported after HDT and autograft in patients with lymphoid malignancies. ${ }^{40-42}$ All patients who developed secondary malignancies had received radiotherapy as part of their first-line therapy or at the time they developed recurrent disease. In addition, it should be noted that four of six secondary malignancies occurred among patients who had to be retreated once or several times for recurrent disease after they received HDS chemotherapy. The absence of total body irradiation in our preparative regimen and the small number of patients who had received MOPP or a MOPP-containing regimen as induction therapy may have reduced their risk of developing secondary malignancy. Nevertheless, a prolonged follow-up will be required to evaluate properly the risk of developing secondary malignancies in this patient population.

The outcome was extremely favorable for the 59 patients who received HDS chemotherapy for their first episode of recurrent disease, with an estimated $63 \%$ EFS rate at 5 years. Similar results were reported recently by the Sloan-Kettering group using an approach analogous to HDS chemotherapy, including intense debulking with two courses of combined ifosfamide, carboplatin, and etoposide followed by HDT with PBPC autograft. ${ }^{18}$ In that single-center study, an estimated $68 \%$ EFS projection was recorded in 56 patients with chemosensitive disease who completed the whole program. Thus, the combined use of intensive debulking with HDT and PBPC autografting seems to produce major improvements in the long-term outcome of patients with nonrefractory HL who require salvage treatment due to failure after induction therapy. The observation that patients who achieve CR prior to autograft had a significantly better outcome compared with patients who did not achieve CR provides further support for the use of intensive chemotherapy as debulking before autograft.

Prolonged disease free survival in patients with HL who experienced their first recurrence after they received combined, intensive debulking and HDT also was observed in a French study. ${ }^{19}$ In that study, the treatment pattern was far from uniform. Approximately $60 \%$ of patients received varying numbers of chemotherapy courses with combined sodium mercaptoethanesulfonate, ifosfamide, novantrone, and etoposide; whereas the remaining patients received several chemotherapy courses. In addition, most patients had a single autograft, generally performed with BEAM; whereas a minority of patients had a tandem autograft, and three patients underwent allogeneic transplantation. Finally, in the French study, treatment feasibility was inadequate, with approximately $40 \%$ of patients unable to complete the final autografting phase. Our program was much more uniform. All 
of our patients underwent the same debulking treatment, and $90 \%$ of patients had the planned single autograft, mostly with the mitoxantrone/L-PAM conditioning regimen. Thus, our observation of prolonged disease free survival in patients at the time they first developed recurrent HL was the result of a well-defined and highly feasible treatment strategy.

The results obtained in patients who had two or more episodes of recurrent disease confirmed the feasibility of using HDS chemotherapy to treat patients after multiple recurrences. A favorable outcome also was observed; although, in these patients, the EFS was not as favorable as the OS. Indeed, despite the promising survival curve, the high incidence of secondary malignancies and the reduced chances of long-term EFS suggest the use of HDS chemotherapy earlier, at the time of the first treatment failure, rather than after multiple recurrences. However, the number of patients in the group with multiple episodes of recurrent disease was too small to draw any definite conclusions.

The benefit of HDS chemotherapy was less evident in the 24 patients who had refractory disease. Low efficacy or no efficacy has been noted in several studies of HDT as salvage treatment for patients with refractory HL. ${ }^{13,14,17,39}$ The 5 -year EFS rate of $33 \%$ observed in our series is among the best results reported to date in these patients who respond poorly. Nevertheless, other approaches should be considered to improve the prognosis of patients with refractory HL. Antibody-based chemoimmunotherapy is among the most promising, novel treatment strategies. ${ }^{43}$ In the alternative, standard or reduced-intensity allogeneic transplantation may be investigated, at least in younger patients who have a human lymphocyte antigen-compatible donor. ${ }^{44,45}$

Presentation with B symptoms also was predictive of a poor outcome in the current study, similar to other reports, ${ }^{10,18,19}$ whereas none of the other clinical features that were proposed as strong predictors of outcome (i.e., age, disease stage, bulky disease, BM or other extralymph node involvement, and duration of first CR) were significantly prognostic. There was no significant difference in OS and EFS between patients with early or late first recurrence, whereas other studies have found a significantly poorer outcome for patients with early recurrence. ${ }^{7,9,12,18}$ Our finding may have been due to the fact that our centers usually perform a restaging 1-2 months after induction. This may imply a strict definition of first CR, with the exclusion of patients who have a transient response and early postchemotherapy disease progression. In the alternative, the potent antitumor activity of HDS chemotherapy may have improved markedly the out- come of patients who developed recurrent disease within 1 year after they achieved CR and abolished their adverse prognosis.

Thus, this multicenter study provides further evidence of the efficacy of HDT as rescue therapy for patients with HL and supports the use of intensive debulking prior to PBPC autografting to maximize its therapeutic benefit. The intensive regimen of bleomycin, etoposide, doxorubicin, cyclophosphamide, vincristine, and prednisone (BEACOP) has been proposed as a very effective induction treatment for patients with high-risk HL. ${ }^{46}$ However, it has not been demonstrated that salvage regimens are as feasible or as effective after BEACOP as they are after less intensive, conventional regimens. To address this issue, a randomized trial of ABVD compared with BEACOP, both coupled with an HDS-like salvage program, in patients who do not respond to induction therapy is underway at IIL centers.

\section{REFERENCES}

1. Goldstone AH, McMillan AK. The place of high-dose therapy with haemopoietic stem cell transplantation in relapsed and refractory Hodgkin's disease. Ann Oncol. 1993;4:21-27.

2. Bierman PJ, Armitage JO. Role of autotransplantation in Hodgkin's disease. Hematol Oncol Clin North Am. 1993;7: 591-611.

3. Armitage JO. Management of relapsed Hodgkin's disease patients with high-dose chemotherapy and marrow stemcell transplantation. Ann Oncol. 2000;11:645-646.

4. Linch DC, Winfield D, Goldstone AH, et al. Dose intensification with autologous bone-marrow transplantation in relapsed and resistant Hodgkin's disease: results of a BNLI randomized trial. Lancet. 1993;341:1051-1054.

5. Chopra R, McMillan AK, Linch DC, et al. The place of highdose BEAM therapy and autologous bone marrow transplantation in poor-risk Hodgkin's disease. a single-center eight-year study of 155 patients. Blood. 1993;81:1137-1145.

6. Yahalom J, Gulati SC, Toia M, et al. Accelerated hyperfractionated total-lymphoid irradiation, high-dose chemotherapy, and autologous bone marrow transplantation for refractory and relapsing patients with Hodgkin's disease. J Clin Oncol. 1993;11:1062-1070.

7. Reece DE, Connors JM, Spinelli JJ, et al. Intensive therapy with cyclophosphamide, carmustine, etoposide \pm cisplatin, and autologous bone marrow transplantation for Hodgkin's disease in first relapse after combination chemotherapy. Blood. 1994;83:1193-1199.

8. Nademanee A, O'Donnell MR, Snyder DS, et al. High-dose chemotherapy with or without TBI followed by ASCT for patients with relapsed and refractory HD: results in 85 patients and analysis of prognostic factors. Blood. 1995;85: 1381-1390.

9. Bierman PJ, Anderson JR, Freeman MB, et al. High-dose chemotherapy followed by autologous hematopoietic rescue for Hodgkin's disease patients following first relapse after chemotherapy. Ann Oncol. 1996;7:151-156. 
10. Horning SJ, Chao NJ, Negrin RS, et al. High-dose therapy and autologous hematopoietic progenitor cell transplantation for recurrent or refractory Hodgkin's disease: analysis of the Stanford University results and prognostic indices. Blood. 1997;89:801-813.

11. Brice P, Bouabdallah R, Moreau P, et al. Prognostic factors for survival after high-dose therapy and autologous stem cell transplantation for patients with relapsing Hodgkin's disease: analysis of 280 patients from the French registry. Bone Marrow Transplant. 1997;20:2--26.

12. Sweetenham JW, Taghipour G, Milligan D, et al. High-dose therapy and autologous stem cell rescue for patients with Hodgkin's disease in first relapse after chemotherapy: results from the EBMT. Bone Marrow Transplant. 1997;20: $745-752$.

13. Lazarus HM, Loberiza FR Jr., Zhang M-J, et al. Autotransplants for Hodgkin's disease in first relapse or second remission: a report from the Autologous Blood and Marrow Transplant Registry (ABMTR). Bone Marrow Transplant. 2001;27:387-396.

14. Sureda A, Arranz R, Iriondo A, et al. Autologous stem-cell transplantation for Hodgkin's disease: results and prognostic factors in 494 patients from the Grupo Español de Linfomas/Transplante Autólogo de Médula Ósea Spanish Cooperative Group. J Clin Oncol. 2001;19:1395-1404.

15. Gianni AM, Siena S, Bregni M, et al. Granulocyte-macrophage colony-stimulating factor to harvest circulating haematopoietic stem cells for autotransplantation. Lancet. 1989;ii:580-585.

16. Schmitz N, Linch DC, Dreger P, et al. Randomised trial of filgrastim-mobilised peripheral blood progenitor cell transplantation versus autologous bone-marrow transplantation in lymphoma patients. Lancet. 1996;347:353-357.

17. Argiris A, Seropian S, Cooper DL. High-dose BEAM chemotherapy with autologous peripheral blood progenitor-cell transplantation for unselected patients with primary refractory or relapsed Hodgkin's disease. Ann Oncol. 2000;11:665672 .

18. Moskowitz CH, Nimer SD, Zelenetz AD, et al. A 2-step comprehensive high-dose chemotherapy second-line program for relapsed and refractory Hodgkin disease: analysis by intent to treat and development of a prognostic model. Blood. 2001;97:616-623.

19. Fermé $C$, Mounier $N$, Diviné $M$, et al. Intensive salvage therapy with high-dose chemotherapy for patients with advanced Hodgkin's disease in relapse or failure after initial chemotherapy: results of the Groupe d'E'tude des Lymphomes de l'Adulte H89 trial. J Clin Oncol. 2002;20:467-475.

20. Gianni AM, Bonadonna G. High dose chemoradiotherapy for sensitive tumors: is sequential better than concurrent drug delivery? Eur J Cancer Clin Oncol. 1989;25:1027-1030.

21. Tarella C, Ferrero D, Bregni M, et al. Peripheral blood expansion of early progenitor cells after high-dose cyclophosphamide and rhGM-CSF. Eur J Cancer. 1991;27:22-27.

22. Gianni AM, Siena S, Bregni M, et al. Prolonged disease-free survival after high-dose sequential chemo-radiotherapy and haemopoietic autologous transplantation in poor prognosis Hodgkin's disease. Ann Oncol. 1991;2:645-653.

23. Gianni AM, Siena S, Bregni M, et al. High-dose sequential chemo-radiotherapy with peripheral blood progenitor cell support for relapsed or refractory Hodgkin's disease-a 6-year update. Ann Oncol. 1993;4:889-891.

24. Carlo Stella C, Cazzola M, De Fabritiis P, et al. CD34-positive cells: biology and clinical relevance. Haematologica. 1995; 80:367-387.

25. Tarella C, Caracciolo D, Corradini P, et al. Long-term follow-up of advanced-stage low-grade lymphoma patients treated upfront with high-dose sequential chemotherapy and autograft. Leukemia. 2000;14:740-747.

26. Cortelazzo S, Rambaldi A, Rossi A, et al. Intensification of salvage treatment with high-dose sequential chemotherapy improves the outcome of patients with refractory or relapsed aggressive non-Hodgkin's lymphoma. $\mathrm{Br}$ J Haematol. 2001;114:333-341.

27. Vitolo U, Liberati AM, Lambertenghi Deliliers G, et al. A multicenter randomized trial by Italian Lymphoma Intergroup (ILI) comparing high dose chemotherapy (HDS) with autologous stem cell transplantation (ASCT) vs. intensified chemotherapy MegaCEOP in high risk diffuse large cell lymphoma (DLCL): no difference in outcome and toxicity. Blood. 2001;98:725a.

28. Ladetto M, Corradini $\mathrm{P}$, Vallet $\mathrm{S}$, et al. High rates of clinical and molecular remissions in follicular lymphoma patients receiving high-dose sequential chemotherapy and autografting at diagnosis: a multicenter, prospective study by the Gruppo Italiano Trapianto Midollo Osseo (GITMO). Blood. 2002;100:1559-1565

29. Harris NL, Jaffe ES, Stein H, et al. A revised EuropeanAmerican classification of lymphoid neoplasms: a proposal from the International Lymphoma Study Group. Blood. 1994;84:1361-1392

30. Lister TA, Crowther D, Sutcliffe SB, et al. Report of a committee convened to discuss the evaluation of staging of patients with HD: Cotswold meeting. J Clin Oncol. 1989;7: 1630-1638.

31. Bonadonna G, Zucali R, Monfardini S, et al. Combination chemotherapy of Hodgkin's disease with Adriamycin, bleomycin, vinblastine and imidazole carboaxamide versus MOPP. Cancer. 1975;36:252-259.

32. Bonadonna G, Valagussa P, Santoro A, et al. Alternating non-cross-resistant combination chemotherapy or MOPP in Stage IV Hodgkin's disease: a report of 8-year results. Ann Intern Med. 1986;104:739-746.

33. Canellos GP, Anderson JR, Propert KJ, et al. Chemotherapy of advanced Hodgkin's disease with MOPP, ABVD, or MOPP alternating with ABVD. N Engl J Med. 1992;327:1478-1484.

34. Gobbi PG, Pieresca C, Ghirardelli ML, et al. Long-term results from MOPPEBVCAD chemotherapy with optional limited radiotherapy in advanced Hodgkin's disease. Blood. 1998;91:2704-2712.

35. Tarella C, Zallio F, Caracciolo D, et al. High-dose mitoxantrone + melphalan (MITO/L-PAM) as conditioning regimen supported by peripheral blood progenitor cell (PBPC) autograft in 113 lymphoma patients: high tolerability with reversible cardiotoxicity. Leukemia. 2001;15:256-263.

36. Kaplan EL, Meier P. Nonparametric estimation from incomplete observation. J Am Stat Assoc. 1958;53:457-481.

37. Mantel N, Haenzel W. Statistical aspects of the analysis of data from retrospective studies of disease. J Natl Cancer Inst. 1958;22:719-723.

38. Cox DR. Regression models and life tables. J R Stat Soc B. 1972;34:187-220.

39. Josting A, Rueffer U, Franklin J, et al. Prognostic factors and treatment outcome in primary progressive Hodgkin lymphoma: a report from the German Hodgkin Lymphoma Study Group. Blood. 2000;96:1280-1286. 
40. André M, Henry-Amar M, Blaise D, et al. Treatment-related deaths and second cancer risk after autologous stem-cell transplantation for Hodgkin's disease. Blood. 1998;92:19331940.

41. Darrington DL, Vose JM, Anderson JR, et al. Incidence and characterization of secondary myelodysplastic syndrome and acute myelogenous leukemia following high-dose chemoradiotherapy and autologous stem-cell transplantation for lymphoid malignancies. J Clin Oncol. 1994;12:2527-2534.

42. Miller JS, Arthur DC, Litz CE, et al. Myelodysplastic syndrome after autologous bone marrow transplantation: an additional late complication of curative cancer therapy. Blood. 1994;83:3780-3786.

43. Huhn M, Sasse S, Tur MK, et al. Human angiogenin fused to human CD30 ligand (Ang-CD30L) exhibits specific cytotoxicity against CD30-positive lymphoma. Cancer Res. 2001;61: 8737-8742.
44. Carella AM, Cavaliere M, Lerma E, et al. Autografting followed by nonmyeloablative immunosuppressive chemotherapy and allogeneic peripheral-blood hematopoietic stem-cell transplantation as treatment of resistant Hodgkin's disease and non-Hodgkin's lymphoma. J Clin Oncol. 2000;18:3918-3924.

45. Corradini P, Tarella C, Olivieri A, et al. Reduced-intensity conditioning followed by allografting of hematopoietic cells can produce clinical and molecular remissions in patients with poor-risk hematologic malignancies. Blood. 2002;99: 75-82.

46. Diehl V, Franklin J, Hasenclever D, et al. BEACOPP, a new dose-escalated and accelerated regimen, is at least as effective as COPP/ABVD in patients with advanced-stage Hodgkin's lymphoma: interim report from a trial of the German Hodgkin's Lymphoma Study Group. J Clin Oncol. 1998;16:3810-3821. 